Research Article

\title{
Building Earthquake Damage Analysis Using Terrestrial Laser Scanning Data
}

\author{
Qisong Jiao (D, Hongbo Jiang, and Qiang Li \\ Institute of Crustal Dynamics, China Earthquake Administration, Beijing 100085, China \\ Correspondence should be addressed to Qisong Jiao; 1985jqs@163.com
}

Received 26 March 2019; Accepted 21 April 2019; Published 23 May 2019

Guest Editor: Tanja K. Šipoš

Copyright (c) 2019 Qisong Jiao et al. This is an open access article distributed under the Creative Commons Attribution License, which permits unrestricted use, distribution, and reproduction in any medium, provided the original work is properly cited.

\begin{abstract}
Terrestrial laser scanners (TLSs) can acquire high-precision three-dimensional point cloud data for earthquake-damaged buildings. In this study, we collected TLS data in the Wenchuan earthquake zone and developed the TLS-BSAM (terrestrial laser scanning-based building shape analysis model) to carry out a building earthquake damage analysis. This model involves equidistance polygon array extraction, shape dispersion parameter calculations, irregular building clustering segmentation, and damage analysis. We chose 21 buildings as samples for the experiments. The results show that when using an equidistance polygon array to depict a three-dimensional building, $0.5 \mathrm{~m}$ is a reasonable sampling interval for building earthquake damage analysis. Using certain characteristic parameters to carry out $K$-means clustering, one can efficiently divide irregular buildings into regular blocks. Then, by weighted averages, the shape dispersion parameters can be calculated to express the damage extent to buildings. Among the shape dispersion parameters, at least the weighted average standard deviations of the tilt direction, rectangularity, compactness, and center point are suitable to reflect the damage extent. Higher values reflect more serious damage. On the basis of existing data, the weighted average standard deviations of the tilt direction and center point can be used to establish discriminant functions that can effectively distinguish the damage extent.
\end{abstract}

\section{Introduction}

With the diversification of remote sensing data and technical progress in data processing, remote sensing technology can now be used to carry out earthquake damage investigations, which has recently become a topic of sustained interest. Compared with traditional field surveys of earthquake damage, earthquake damage assessments based on remote sensing data are faster, more efficient, and safer. A wide range of earthquake-induced building damage information can be quickly acquired and assessed with new remote sensing technologies. Under the guidance of mathematical statistics, objectoriented methods, and fractal theory, different types of remote sensing data such as optical images, synthetic aperture radar (SAR) imagery, and airborne LiDAR data have been used in building damage assessments. Saito et al. [1] used optical images with $1 \mathrm{~m}$ ground resolution taken by IKONOS before and after the Gujarat earthquake of January 26, 2001, to extract the information about collapsed buildings. Stramondo et al. [2] analyzed the capability of satellite radar and optical remote sensing for earthquake damage detection in urban areas and explored the combined use of radar (SAR) and optical satellite data. Balz and Liao [3] used postseismic high-resolution SAR satellite data for building damage detection caused by the Wenchuan earthquake. Based on airborne LiDAR data, Lin et al. [4] proposed a multiple-primitive-based TIN progressive densification point cloud filtering method to distinguish the ground points and nonground points. $\mathrm{He}$ et al. [5] used an original three-dimensional shape descriptor to detect roofs with surface damage and roofs exhibiting structural damage by identifying spatial patterns of compact and regular contours for intact roofs. Li et al. [6] used LiDAR data and high-resolution optical images to detect damages on the scale of a building's 
rooftop area and realized a quantitative estimation of the degree of building damage. These studies have important significance for facilitating rapid responses during earthquake relief and postearthquake reconstruction work.

Various remote sensing technologies were employed after the Wenchuan earthquake [7-9], and these data are valuable for damage assessment. However, as the aforementioned studies illustrate, earthquake damage assessment based on remote sensing data also has some disadvantages. Specifically, only information about the top surface and part of a building's sides can be recorded by optical remote sensing data. In addition, earthquake damage assessments based on remote sensing data have been generally aimed at large areas containing many buildings and not a single building. Based on earthquake emergency work of recent years in China, we found that optical remote sensing data cannot identify specific building structures that have been destroyed but have top surfaces that are basically intact. Thus, the accuracy of remote sensing-based damage assessment technology is relatively low and field survey data are still needed to carry out earthquake relief and reconstruction work.

As an alternative data collection method, terrestrial laser scanners (TLSs) can be employed from remote locations. A TLS is a fully automated instrument capable of acquiring high-precision three-dimensional scanning data. It emits a high-speed laser that performs noncontact scanning measurements, and three-dimensional coordinates of the target stored in point cloud format are obtained [10]. This technology is especially useful for carrying out measurements in complex and high-risk environments. Furthermore, TLS technology is advantageous because of its ease of operation with high degrees of automation compared with airborne LiDAR. Point cloud data combined with computer-aided design software can quickly be used to rebuild the target's shape and obtain its three-dimensional digital model. To date, TLS technology has been shown to be a powerful tool in studies involving vegetation mapping, geological hazard control, historic preservation, etc. [11-14]. With regard to earthquake damage assessments, TLSs can provide high-precision three-dimensional information about damaged building structures. Although some scholars have employed TLSs in field survey work after earthquakes and tsunamis and have acquired point cloud data for damaged buildings and secondary disaster areas $[15,16]$, studies on the quantitative extraction and analysis of building damage information from point cloud data are insufficient.

To solve this problem, we carried out measurements using a TLS in the Wenchuan earthquake zone and proposed the use of the TLS-BSAM (terrestrial laser scanning-based building shape analysis model), which is based on the theory of shape analysis, to carry out quantitative extraction and analysis of building damage information. This model fully considers building irregularities after earthquake damage, and this study represents a useful attempt to provide important basic data for building damage pattern analysis and simulation.

\section{Methods}

2.1. Shape Analysis. Shape analysis involves storing the shape feature information extracted from images in a particular data structure to carry out identification, comparison, retrieval, and classification procedures $[17,18]$. The main steps of shape analysis are computer-driven and include data preprocessing, feature extraction, and shape classification. Data preprocessing is the basis of shape analysis. The purpose of this step is to obtain the outline or surface structure of the object's shape. These data have a great influence on the performance of the shape feature extraction algorithm. Shape feature extraction is the key of shape analysis. Its purpose is to find an accurate and feasible shape representation. Shape classification involves comparing and statistically computing the similarity of object characteristic factors and then identifying unknown samples according to existing examples. The national standard (classification of earthquake damage to buildings and special structures (GBT 24335-2009)) points out that the classification principles for earthquake-damaged buildings are mainly based on loadbearing components with some consideration of non-loadbearing components. In addition, the degrees of difficulty of repair and function loss are also taken into account. In earthquake field surveys, technicians evaluate the damage level based on a building's cracks and assess the degree of dislocation, deformation of walls, integrity of roofs, structural poles, etc. This is a shape analysis process, which provides us with a theoretical basis to use TLS point cloud data while carrying out earthquake damage extraction and analysis.

2.2. Building Dimension Reduction from Three to Two Dimensions. Unlike plane data, point cloud data record different types of building damage in a three-dimensional space. The existing edge detection algorithm for a plane cannot yet effectively build functions to describe a building's three-dimensional contour or area. To simplify and effectively express the shape of a three-dimensional building by means of "dimension reduction," as shown in Figure 1, we used a set of evenly spaced parallel sections to cut the building's point cloud data and acquire intersecting lines that reflect the shape changes at different heights. This transforms the three-dimensional surface recognition into two-dimensional polygon shape (hereafter called the "equidistance polygon array" or EPA) recognition features.

The extraction of EPA data is an important part of data preprocessing in the TLS-BSAM, and the main procedure involves extracting the point cloud contour lines. The edge detection and extraction algorithms for two-dimensional images are quite mature, but they are not suitable for a discrete point cloud. We thus used the convex hull algorithm to solve this problem. The convex hull algorithm can find the minimum convex polygon that meets the condition that all points are inside the polygon or on the edge $[19,20]$.

Buildings are built in accordance with designs and structural plans, and they are orderly entities, not randomly self-organizing systems. Thus, an intact building with a 


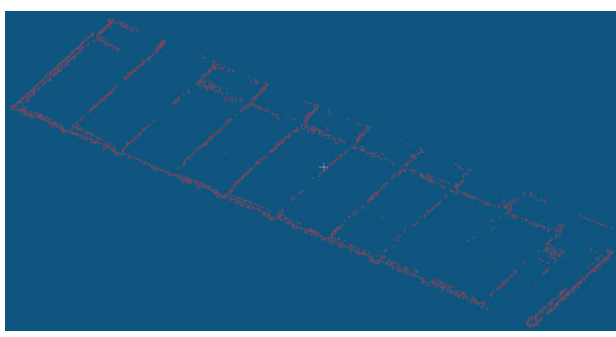

(a)

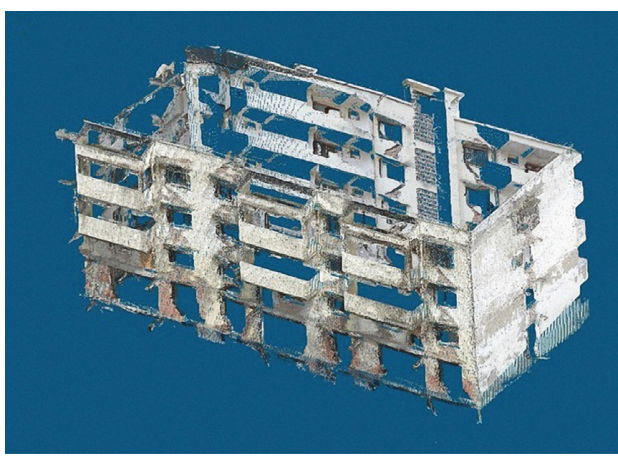

(c)

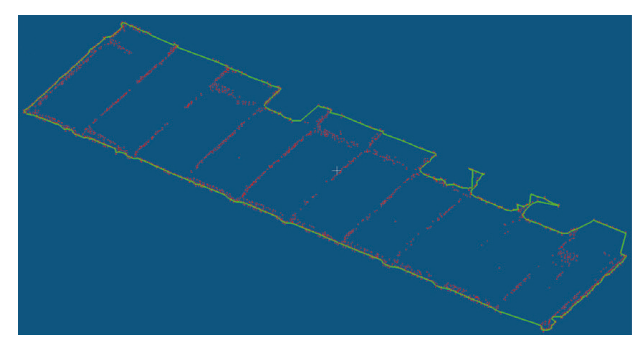

(b)

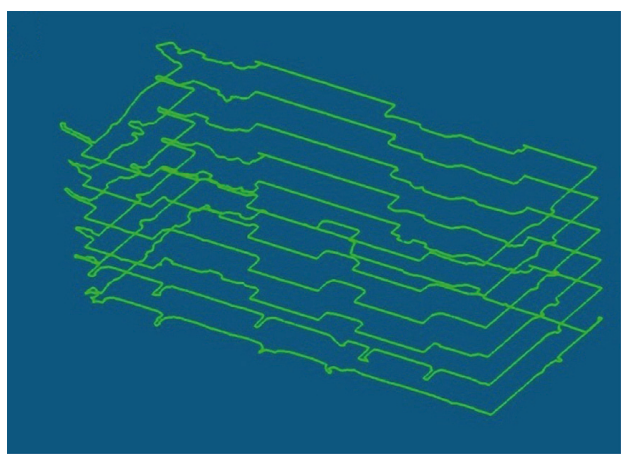

(d)

FIGURE 1: Sketch of a building's "dimension reduction." (a) One layer of the building's point cloud; (b) the layer's convex hull polygon; (c) the building's real point cloud; (d) the building's equidistance polygon array at $2 \mathrm{~m}$ intervals.

regular shape has a very high similarity EPA and the dispersion of shape characteristic parameters is small. In areas with earthquake-damaged buildings, these orderly systems are destroyed. Consequently, equidistance polygons at different heights will show different degrees of deformation, which ultimately results in a dispersion increase for the shape characteristic parameters. In this study, the analysis of building earthquake damage is based on the degree of shape characteristic parameters dispersion in the EPA. Specifically, building damage is proportional to the degree of shape characteristic parameters dispersion, which means that higher dispersion degrees are associated with greater extents of damage. The standard deviation was used to describe the dispersion degree. Here, larger values of standard deviation are associated with higher dispersion degrees and greater extents of damage.

\subsection{Shape Characteristic Parameters Selection. The EPA is} made up of the shape polygons, so we chose the shape characteristic parameters based on contour lines to describe its shape, as shown in Table 1.

In practice, the shape characteristic parameters selection does not adhere to "the more the better" principle. Circumference and area are not prominent when describing the shape in some situations. Thus, we chose the aspect ratio $r$, tilt direction $\theta$, rectangularity $R$, and compactness $C$ as the main shape characteristic parameters. The compactness $C$ reflects the border regularity, and larger $C$ values are associated with more complex shapes. Meanwhile, we noticed during earthquake field surveys that, although buildings were almost intact, they were already tilted. Therefore, we used the polygon central point $(x, y)$ as another shape characteristic parameter. If the central point deviation is large, building damage should not be ignored even if the degree of dispersion for the other shape characteristic parameters is small.

2.4. Irregular Building Clustering Segmentation. A building's three-dimensional shape is sometimes irregular, as shown in Figure 2. For such buildings, the standard deviation $\sigma$ of shape characteristic parameters is often large even if the buildings are intact. To solve this problem, we used the $K$ means clustering algorithm to divide irregular buildings into several regular parts, which involved dividing the EPA into several regular polygon sequences.

When clustering is completed, the polygon number of different parts is the weight in the earthquake building damage analysis, and larger numbers are associated with higher weights. The building shape dispersion parameters are their weighted average standard deviations.

It should be pointed out that $m$ is the clustering number of the EPA and is identified by the investigator according to the building situation uncovered during the field investigation. When the buildings up-down structures are identical or completely collapsed, the $m$ value is equal to one.

2.5. Earthquake Damage Analysis for Buildings. The Chinese seismic intensity scale (GBT 17742-2008) divides earthquake-damaged buildings into five grades, namely, those that are completely destroyed, seriously damaged, moderately (medium) damaged, slightly damaged, and basically undamaged. In this standard, the main difference 
TABle 1: Common shape characteristic parameters.

\begin{tabular}{lcc}
\hline Name & Expression & Description \\
\hline Area & $A$ & Polygon area \\
Circumference & $P$ & Polygon circumference \\
Length & $l$ & Length of the minimum enclosing rectangle (MER) \\
Width & $w$ & Width of the MER \\
Aspect ratio & $r=l / w$ & Length and width ratio of the MER \\
Tilt direction & $\theta$ & Included angle for long and polar axes of the MER \\
Rectangularity & $R=A / A_{\mathrm{MER}}$ & Area ratio of the polygon and its MER \\
Compactness & $C=P^{2} / A$ & Circumference square and area ratio of the polygon \\
\hline
\end{tabular}

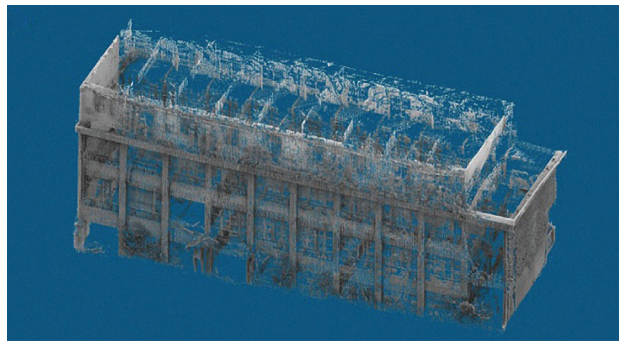

(a)

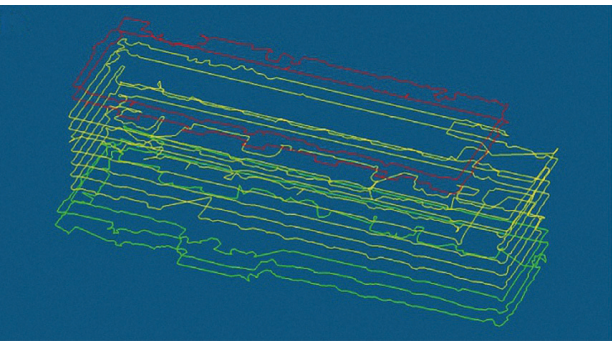

(b)

Figure 2: A building's point cloud and its EPA map. (a) Building with three different blocks; (b) the building's corresponding EPA in three colors.

between essentially undamaged and slightly damaged buildings is the fracture scale of load-bearing components. This is an endpoint that even experienced investigators often have trouble assessing correctly. Here, we merged these two grades into the slightly damaged grade. In view of the existing technology based on remote sensing data, we are able to easily identify completely destroyed buildings, and thus, we are no longer using point cloud data to analyze them. According to the Earthquake Disaster Standards of the People's Republic of China (DB/T75-2018) "Earthquake Disaster Remote Sensing Assessment Part 3: Building damage," the damage grades of single building are divided into three types including collapse, partial collapse, and no collapse. Among them, the partial collapse is defined as the collapse of part of the building, or the partial destruction of the roof, or the destruction of the surrounding wall. The collapse is defined as the roof completely collapses, or more than $50 \%$ of the main structure collapses, twists, deforms, or tilts. Here, we refer to this standard and the serious damage corresponds to collapse and medium damage corresponds to partial collapse.

In this study, the core of the seismic damage analysis is based on a set of known samples with specific characteristic parameters correlated with the earthquake damage; these relationships then serve as a guide for the earthquake damage assessments of unknown buildings. Discriminant analysis is a good technique to accomplish this task. We took the building shape dispersion parameters as the discriminant factors and used Fisher's discriminant method to calculate the linear discrimination functions that were applied to identify the earthquake damage.
Based on the above procedures, we developed the TLSBSAM to carry out the building earthquake damage analysis. The main workflow of the model is shown in Figure 3.

\section{Experiment}

The Wenchuan Ms8.0 earthquake occurred on May 12, 2008, and was the most destructive earthquake recorded since the establishment of the People's Republic of China. The epicenter was located at $31.01^{\circ} \mathrm{N}, 103.42^{\circ} \mathrm{E}$. The earthquake affected most of China including Gansu, Qinghai, Ningxia, Shanxi, Shanxi, Shandong, Henan, Hunan, Hubei, and Chongqing with an area over $100,000 \mathrm{~km}^{2}$. The causative fault was the Longmen mountain fault zone on the eastern margin of the Tibetan Plateau with a focal depth of $14 \mathrm{~km}$. Due to the rapid uplift of the Tibetan Plateau and the influence of gravity, the eastern margin of the Tibetan Plateau gradually subducted along the Longmen mountains and was meanwhile blocked by the Sichuan Basin, resulting in longterm accumulation of tectonic stress. The final stress was suddenly released in the Beichuan to Yingxiu area of Longmen Mountain, triggering a right-lateral thrust earthquake. The epicenter intensity of the Wenchuan earthquake was as high as 11 and the destructiveness was huge. In 2013, Beichuan and Hanwang, which are located in close proximity to the epicenter, were protected as earthquake sites. Buildings were seriously damaged in both of these areas. We selected these two sites as the study area and used a Riegl VZ-1000 laser scanning system equipped with the Riscan Pro software (Figure 4) to acquire and process the building point cloud data. 


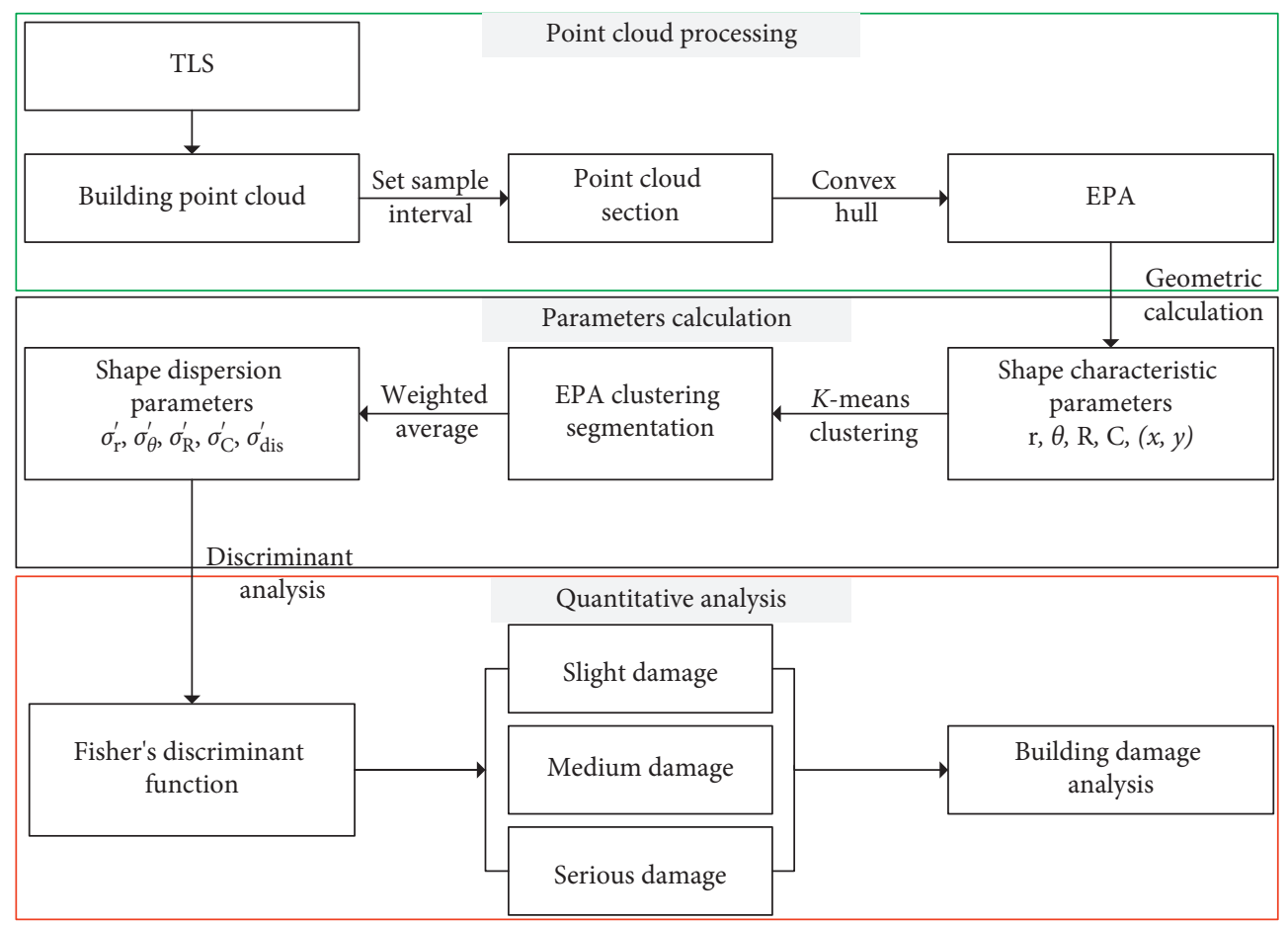

FIgURE 3: Flow chart of the TLS-BSAM.

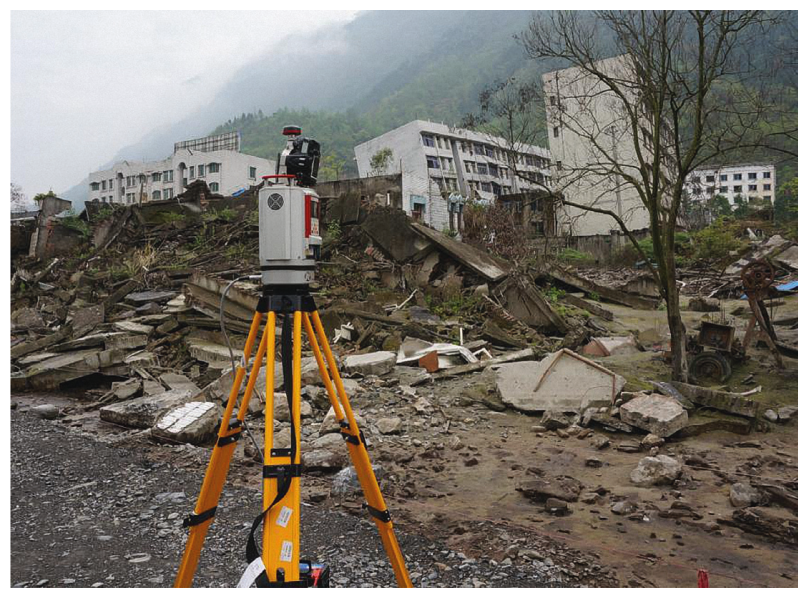

FIgURE 4: Riegl VZ-1000 laser scanning system.

Prior to the start of the scanning work, a field reconnaissance survey must be conducted to confirm the main objects in the scanning area based on the buildings' original layout. The buildings' locations, areas, and shapes are all taken into consideration when determining the scanning station sites. In addition, to meet the requirements of data registration, each station must have a certain amount of overlap. The accuracy of the scanner is $8 \mathrm{~mm}$ at $100 \mathrm{~m}$, and all scans in this study were performed at a close range $(<40 \mathrm{~m}$ from the target), which helps to improve accuracy. During data registration, the registration error between adjacent scans was less than $2 \mathrm{~mm}$. After all scans were aligned using the Riscan Pro software, the root mean square (RMS) error was less than $7 \mathrm{~mm}$. Following the measurement, building samples were picked from these datasets and each building was isolated to eliminate noise. The station distribution and the data processing results are shown in Figure 5.

\section{Results and Discussion}

We selected 21 buildings to build the sample dataset, and these structures included 13 damaged buildings in Beichuan and 6 in Hanwang. In order to compare baseline data with the damaged buildings, we also scanned two intact buildings in Dujiangyan and Beijing. The building sample information from the field survey is shown in Table 2. On the basis of the TLS-BSAM, we calculated the $\sigma_{\mathrm{r}}^{\prime}, \sigma_{\theta}^{\prime}, \sigma_{\mathrm{R}}^{\prime}, \sigma_{\mathrm{C}}^{\prime}$, and $\sigma_{\text {dis }}^{\prime}$ for these 21 buildings and analyzed them with regard to the four aspects discussed in the following sections.

4.1. Influence of Different Sample Intervals on Shape Dispersion Parameters. In order to identify the influence of different sample intervals on shape dispersion parameters, we evaluated six different sample intervals set at $0.25 \mathrm{~m}, 0.5 \mathrm{~m}$, $1 \mathrm{~m}, 1.5 \mathrm{~m}, 2 \mathrm{~m}$, and $2.5 \mathrm{~m}$. With these increases in the sampling intervals, the shape dispersion parameters changed showing a similar behavior. Taking buildings B12, B14, H2, and $\mathrm{H} 3$ as examples (Figure 6), the extent of change for $\sigma_{\theta}^{\prime}$ was relatively small for sampling intervals of 0.25 to $1.0 \mathrm{~m}$ and $\sigma_{\mathrm{C}}^{\prime}$ was also relatively small within this range.

This result indicates that when the sample interval is too large, not enough of the EPA can be extracted to reflect building damage features smaller than the sample interval. In addition, not enough samples are collected for clustering, which leads to increases in the error of the shape dispersion parameters. When the sample interval is greater than $2 \mathrm{~m}$, it is close to the height of most buildings and thus not suitable 

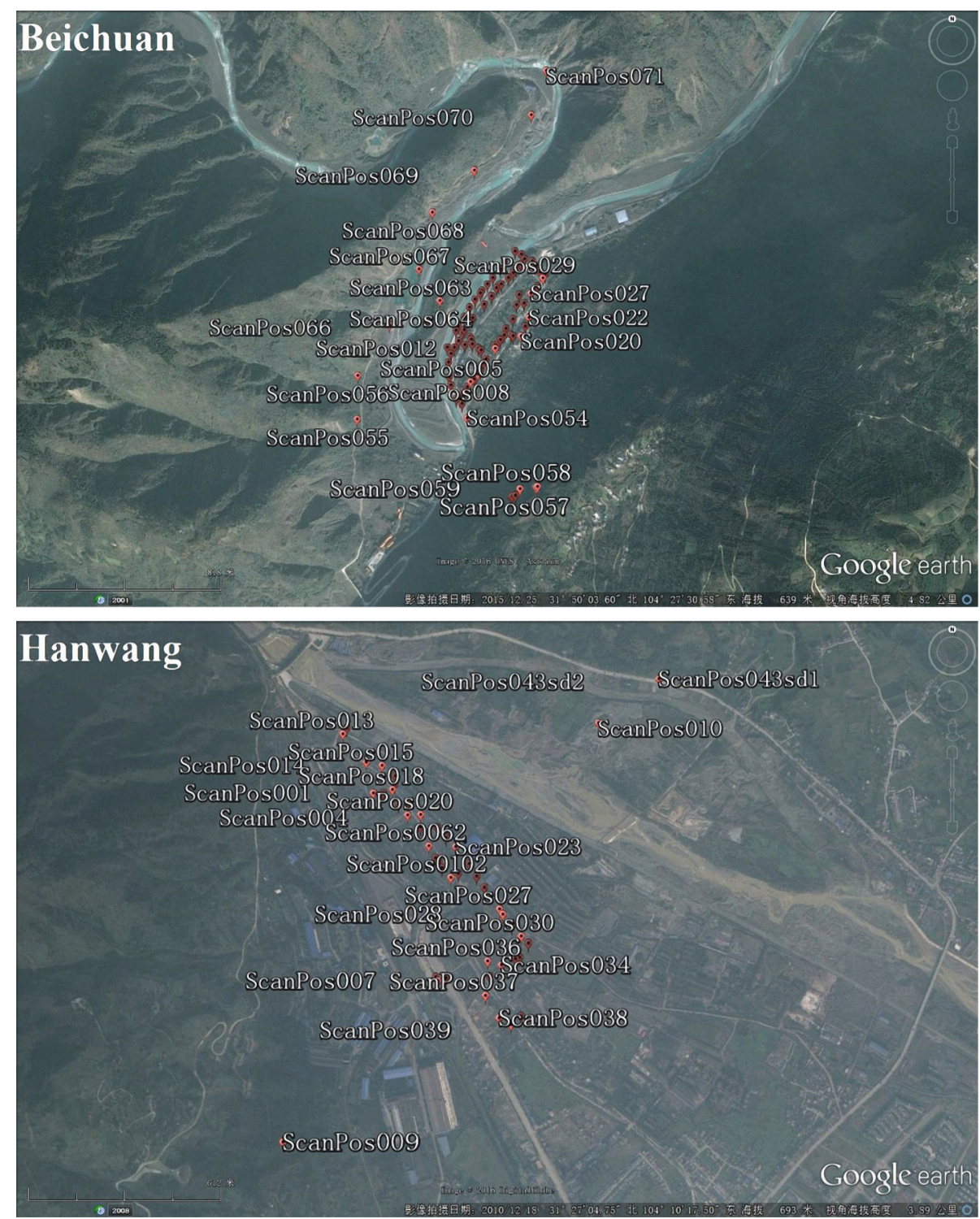

(a)
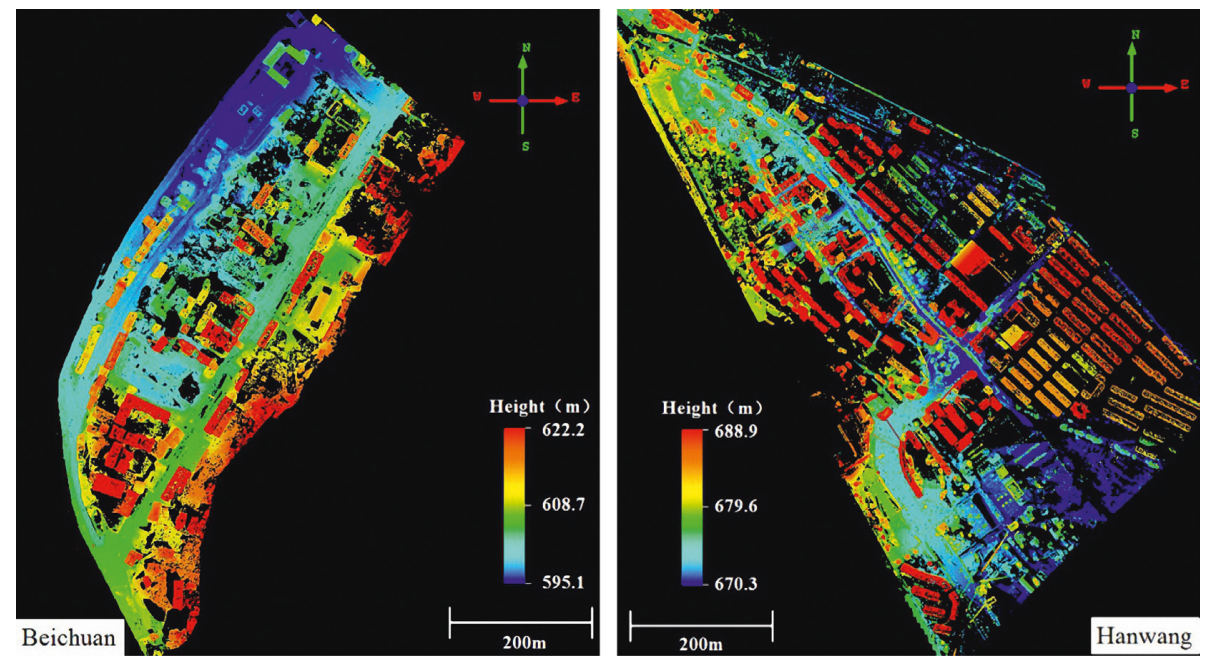

(b)

Figure 5: Station distribution (a) and point cloud top view map (b) of Beichuan and Hanwang. 
TABLE 2: Sample information of the buildings.

\begin{tabular}{|c|c|c|c|c|c|}
\hline ID & Location & Height $(\mathrm{m})$ & Block & Structural system & Damage degree \\
\hline $\bar{B} 1$ & \multirow{13}{*}{ Beichuan } & 16.67 & 3 & Masonry building & Medium \\
\hline $\mathrm{B} 10$ & & 14.46 & 3 & $\mathrm{RC}$ frame & Medium \\
\hline B11 & & 24.42 & 1 & RC frame & Slight \\
\hline $\mathrm{B} 12$ & & 21.40 & 2 & $\mathrm{RC}$ frame & Medium \\
\hline B14 & & 14.32 & 3 & $\mathrm{RC}$ frame & Medium \\
\hline B16 & & 15.81 & 1 & Masonry building & Serious \\
\hline B2 & & 24.02 & 2 & RC frame & Medium \\
\hline B3 & & 13.48 & 1 & Masonry building & Medium \\
\hline B4 & & 24.35 & 2 & RC frame & Serious \\
\hline B6 & & 21.53 & 1 & $\mathrm{RC}$ frame & Slight \\
\hline B7 & & 20.80 & 2 & $\mathrm{RC}$ frame & Slight \\
\hline B8 & & 22.37 & 1 & $\mathrm{RC}$ frame & Serious \\
\hline B9 & & 12.69 & 2 & Masonry building & Serious \\
\hline D1 & Dujiangyan & 24.14 & 5 & RC frame & Intact \\
\hline $\mathrm{H} 1$ & \multirow{6}{*}{ Hanwang } & 16.07 & 2 & RC frame & Serious \\
\hline $\mathrm{H} 2$ & & 18.27 & 2 & $\mathrm{RC}$ frame & Medium \\
\hline $\mathrm{H} 3$ & & 9.81 & 2 & Masonry building & Serious \\
\hline $\mathrm{H} 4$ & & 19.78 & 3 & RC frame & Slight \\
\hline $\mathrm{H} 8$ & & 13.39 & 2 & Masonry building & Slight \\
\hline $\mathrm{H} 9$ & & 27.70 & 3 & RC walls & Slight \\
\hline J1 & Beijing & 29.37 & 7 & RC walls & Intact \\
\hline
\end{tabular}

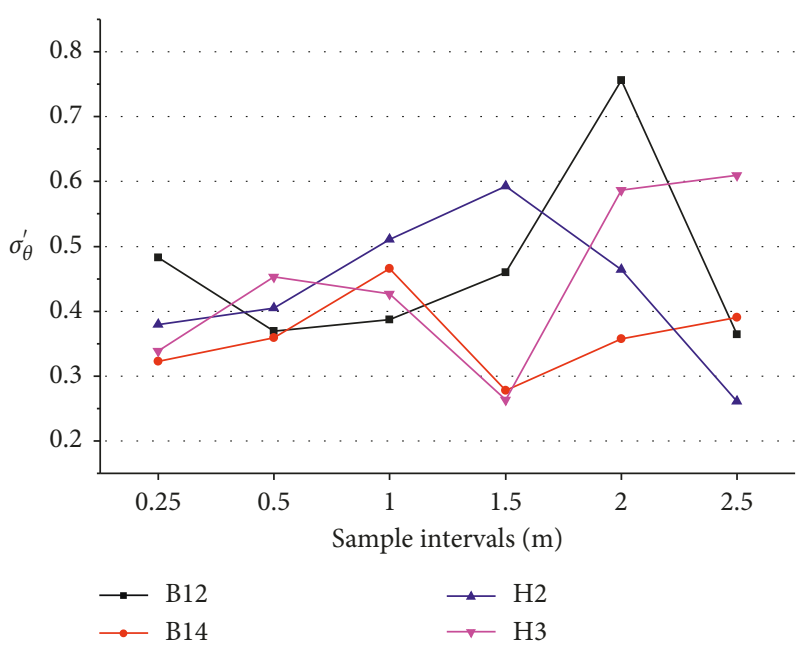

(a)

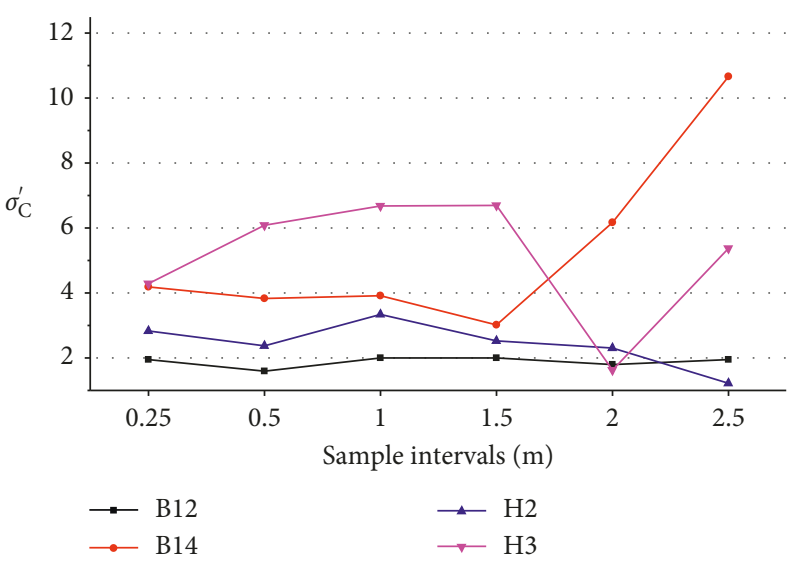

(b)

Figure 6: Plot of the response of $\sigma_{\theta}^{\prime}$ (a) and $\sigma_{\mathrm{C}}^{\prime}(\mathrm{b})$ to different sample intervals.

for shape analysis. However, small sample intervals will increase the error of the shape dispersion parameters because of noise within the point cloud. After experimenting with the data, $0.5 \mathrm{~m}$ was selected as the ideal sample interval.

4.2. Clustering Segmentation Results for Irregular Buildings. The TLS-BSAM divides irregular buildings using the $K$ means clustering method, which makes the shape dispersion parameters truly reflect the extent of building damage. For this analysis, buildings B8 and H4 were used as examples (Figure 7); B8 is a regular building that was badly damaged, while $\mathrm{H} 4$ is an irregular building that was slightly damaged.

In order to test the clustering results of the TLS-BSAM, we calculated the shape dispersion parameters of $\mathrm{H} 4$ before (1 block) and after (3 blocks) clustering (Table 3 ).

As shown in Figure 8, the $\sigma_{\mathrm{r}}^{\prime}, \sigma_{\theta}^{\prime}, \sigma_{\mathrm{R}}^{\prime}, \sigma_{\mathrm{C}}^{\prime}$, and $\sigma_{\text {dis }}^{\prime}$ values for $\mathrm{H} 4$ before clustering were greater than the corresponding values after clustering, and the $\sigma_{\mathrm{r}}^{\prime}, \sigma_{\theta}^{\prime}, \sigma_{\mathrm{C}}^{\prime}$, and $\sigma_{\text {dis }}^{\prime}$ values were even greater than the values for B8, which was badly damaged. However, after clustering, the shape dispersion parameters for $\mathrm{H} 4$ were all smaller than those for B8. In other words, the clustering method helped the parameters to reflect the real building damage. 


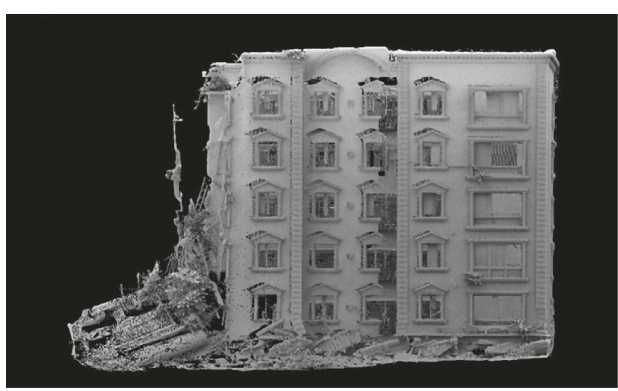

(a)

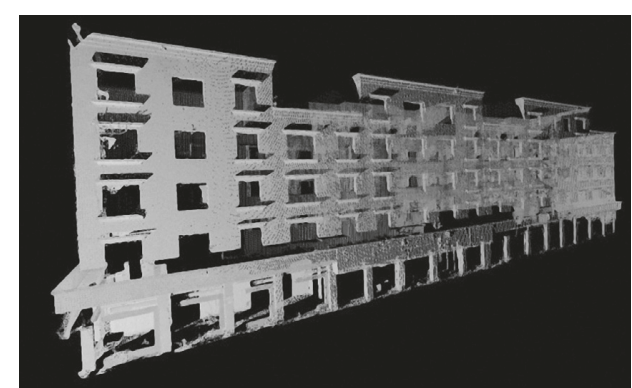

(b)

Figure 7: Buildings with different degrees of earthquake-induced damage. (a) B8 is a regular building that was badly damaged, while (b) H4 is an irregular building that was slightly damaged.

TABLE 3: Shape dispersion parameters of B8 and H4.

\begin{tabular}{lccccccc}
\hline ID & Damage degree & Block & $\sigma_{\mathrm{r}}^{\prime}$ & $\sigma_{\theta}^{\prime}$ & $\sigma_{\mathrm{R}}^{\prime}$ & $\sigma_{\mathrm{C}}^{\prime}$ & $\sigma_{\text {dis }}^{\prime}$ \\
\hline B8 & Serious & 1 & 0.038 & 0.471 & 0.123 & 4.232 & 0.784 \\
\multirow{2}{*}{$\mathrm{H} 4$} & \multirow{2}{*}{ Slight } & 3 & 0.027 & 0.286 & 0.027 & 1.358 & 0.349 \\
& & 1 & 0.206 & 0.545 & 0.074 & 13.349 & 1.245 \\
\hline
\end{tabular}

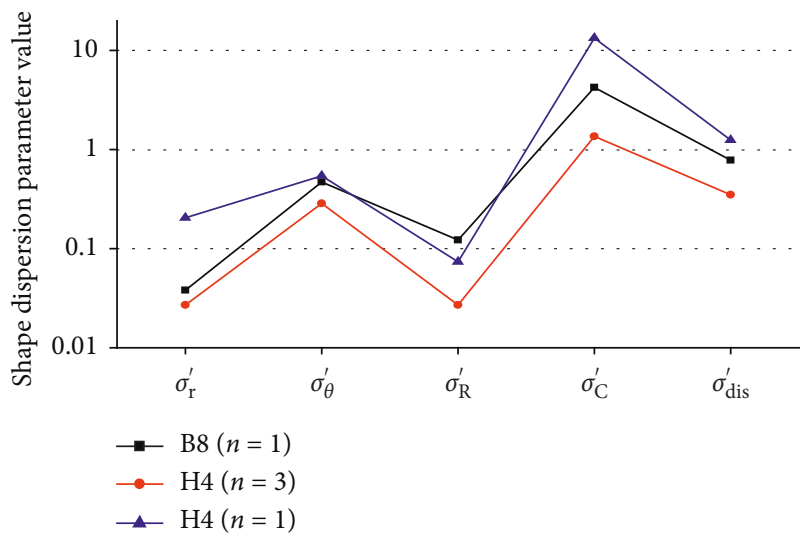

FIGURE 8: Shape dispersion parameter comparison plot before ( $\mathrm{H} 4$ and $\mathrm{B} 8, n=1)$ and after $(\mathrm{H} 4, n=3)$ clustering for the badly damaged (B8) and slightly damaged (H4) buildings.

\subsection{Comparative Analysis of Shape Dispersion Parameters of} Buildings Damaged to Different Extents. By comparing the parameters of buildings damaged to different extents (Figure 9), it can be seen that slightly damaged buildings generally have low values, while seriously damaged buildings have high values and medium damaged buildings have medium values. Only one exception to this trend was detected, i.e., the $\sigma_{\mathrm{r}}^{\prime}$ value of seriously damaged buildings was not significantly higher than that of medium damaged buildings. This suggests that at least $\sigma_{\theta}^{\prime}, \sigma_{\mathrm{R}}^{\prime}$, $\sigma_{\mathrm{C}}^{\prime}$, and $\sigma_{\mathrm{dis}}^{\prime}$ values are able reflect the extent of building damage.

In order to compare the different parameters, we drew box plots (Figure 10). For a given parameter, flatter boxes are indicative of more concentrated values. In addition, the larger the boxes distance from the horizontal axis, the greater the difference in the extent of damage between buildings. If a parameter can meet both conditions at the same time, it suggests that this parameter can better reflect the extent of damage to the buildings.

As shown in Figure 10, except for $\sigma_{\mathrm{r}}^{\prime}$, the values of the other four parameters change from large values to small values in correspondence with the changes in the extent of damage from serious to slight. In other words, the greater the value, the more extensive the damage. The larger boxes of $\sigma_{\mathrm{C}}^{\prime}$ and $\sigma_{\text {dis }}^{\prime}$ allow for distinguishing the extent of the damage.

4.4. Discriminant Analysis of a Building's Damage Extent. A multifactor analysis method is needed to express the relationship between the shape dispersion parameters and the damage extent to buildings. Here, based on the existing sample data, we used Fisher's discriminant analysis method to obtain the linear discrimination functions relevant to the damage extent. According to the statistical results in Table 4, the parameters that can effectively identify the buildings' damage extent are $\sigma_{\mathrm{C}}^{\prime}$ and $\sigma_{\text {dis }}^{\prime}$, while the other parameters were eliminated from the analysis because they show values of $F \leq 2.71$.

Fisher's linear discrimination functions are shown as follows:

(i) Slight damage: $F 1=6.528 \sigma_{\mathrm{C}}^{\prime}+17.956 \sigma_{\mathrm{dis}}^{\prime}-9.761$.

(ii) Medium damage: $F 2=9.675 \sigma_{\mathrm{C}}^{\prime}+27.530 \sigma_{\text {dis }}^{\prime}-20.560$.

(iii) Serious damage: $F 3=16.961 \sigma_{\mathrm{C}}^{\prime}+41.302 \sigma_{\text {dis }}^{\prime}-55.945$.

By substituting the $\sigma_{\mathrm{C}}^{\prime}$ and $\sigma_{\text {dis }}^{\prime}$ values into the three functions above, the buildings' damage extent was classified into respective categories according to the maximum values obtained, i.e., if the maximum value was obtained with the slight damage function, then that building was classified as slightly damaged. According to the space distribution scatter plots (Figure 11), the three damage extent centers were far apart and the sample points within the same damage extent area were relatively concentrated. One medium damaged building was wrongly classified as slightly damaged, and one seriously damaged building was wrongly classified as medium damaged. The discriminant results were generally accurate.

In summary, among all the parameters discussed above, $\sigma_{\mathrm{C}}^{\prime}$ and $\sigma_{\text {dis }}^{\prime}$ are best suited for use in combination to distinguish the extent of building damage of the existing building samples. These findings prove that the proposed 


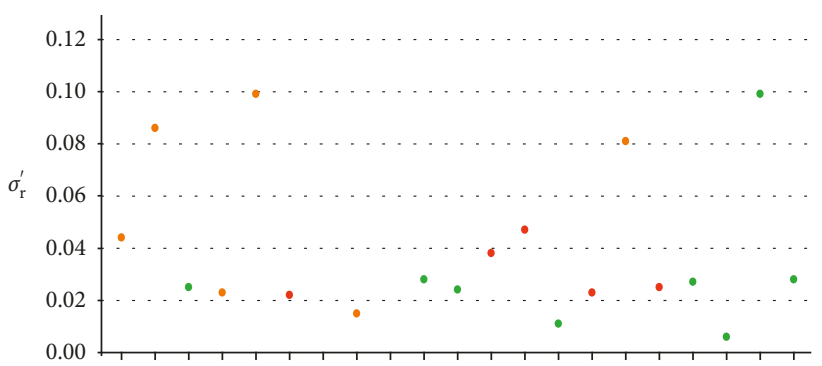

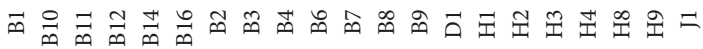

ID

- Serious damage

- Medium damage

- Slight damage

(a)

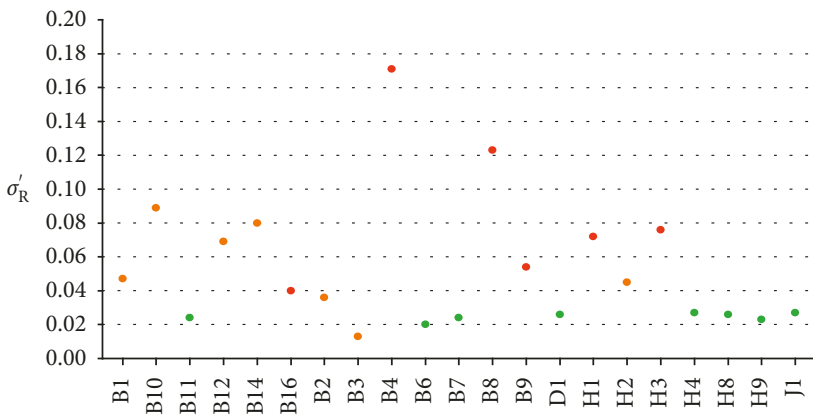

ID

- Serious damage

- Medium damage

- Slight damage

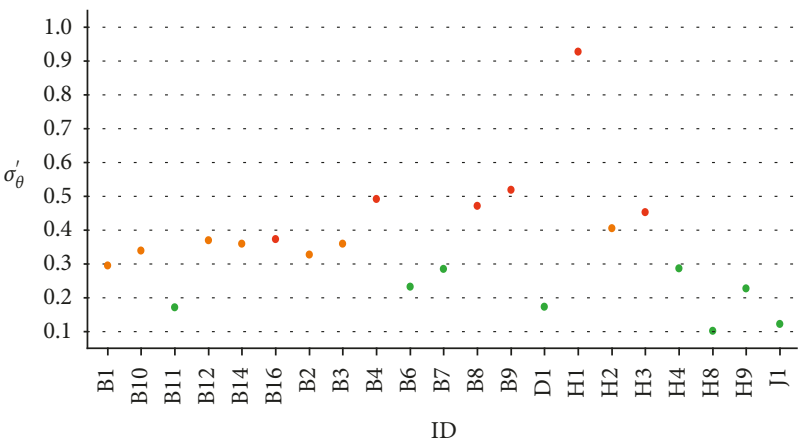

- Serious damage

- Medium damage

- Slight damage

(b)

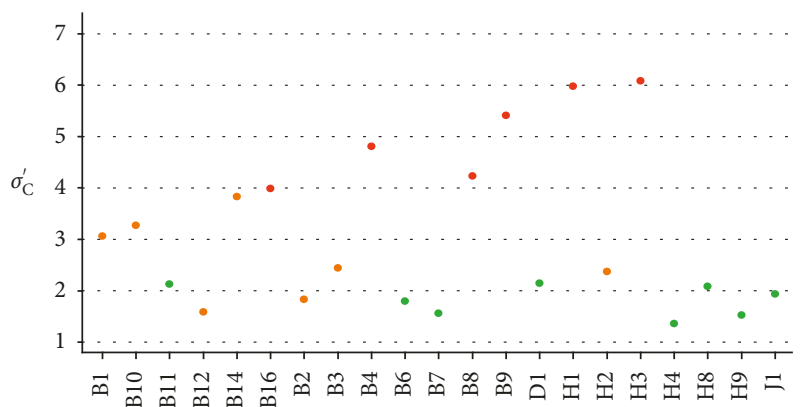

ID

- Serious damage

Medium damage

- Slight damage

(c)

(d)

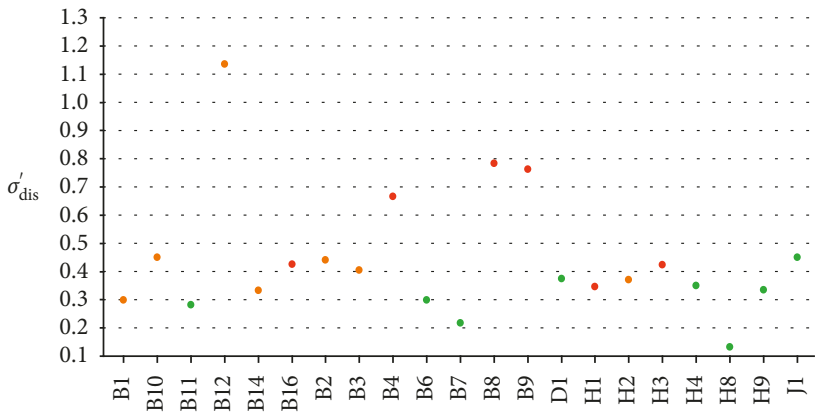

ID

- Serious damage
- Sedium damage
- Slight damage

(e)

FiguRe 9: Shape dispersion parameter comparison plots for different buildings.

TLS-BSAM is capable of extracting and analyzing building earthquake damage extents.

\section{Conclusions}

In order to realize building earthquake damage analysis based on TLS point cloud data, TLS-BSAM was proposed. This model is based on shape analysis theory, which solves the problems of EPA extraction, shape dispersion parameter calculations, irregular building clustering segmentation, and damage analysis. We used 21 buildings as samples to carry out our experiments. The main conclusions are as follows:

(1) The "dimension reduction" method, which uses the EPA to express three-dimensional building data, is an effective way to carry out shape analysis, and $0.5 \mathrm{~m}$ is a reasonable sampling interval for the EPA during building earthquake damage analysis. 


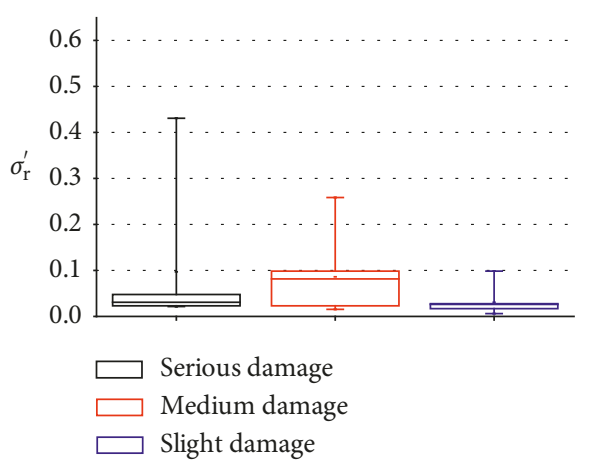

(a)

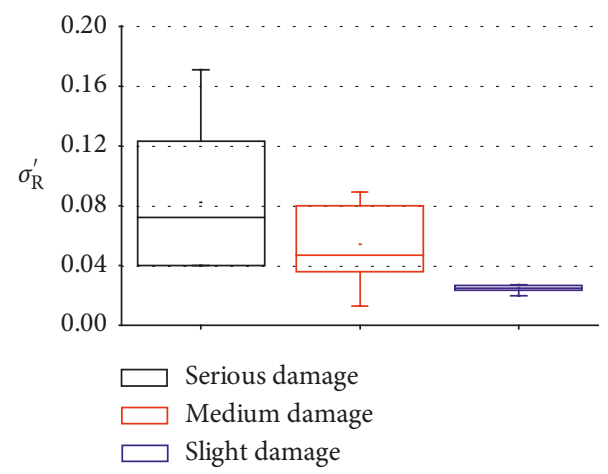

(c)

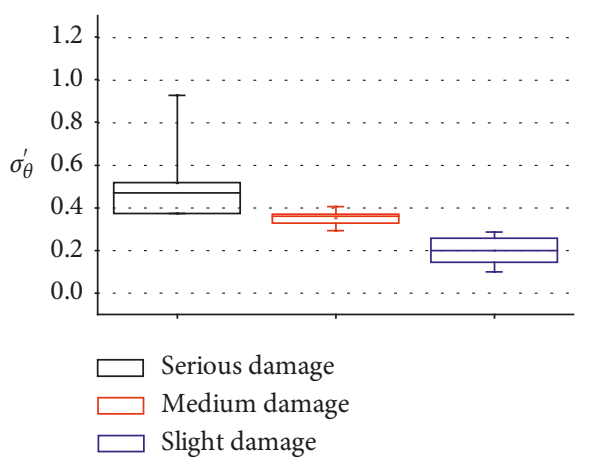

(b)

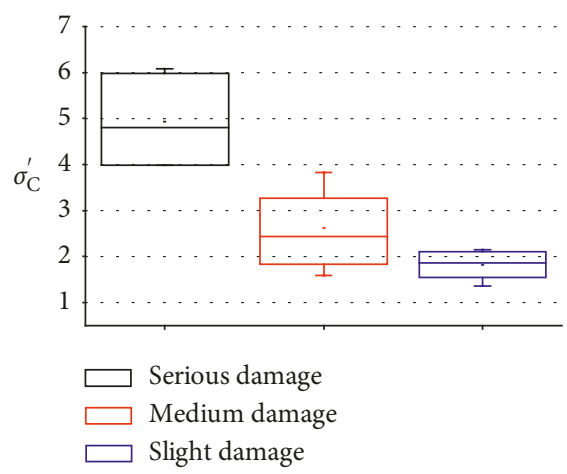

(d)

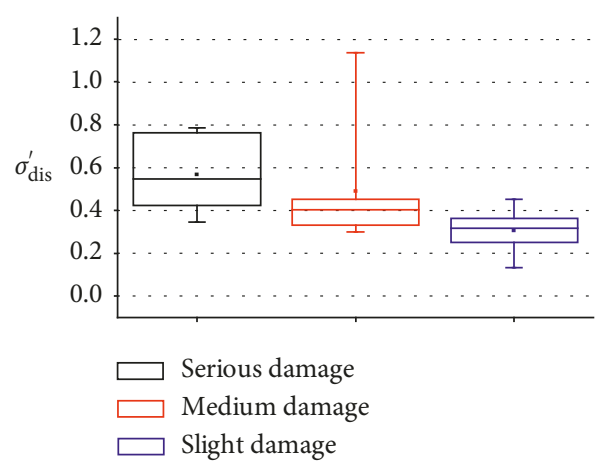

(e)

Figure 10: Shape dispersion parameter box plots.

TABLE 4: Statistical results for selection of different variables.

\begin{tabular}{lccc}
\hline Variable & Tolerance & $F$ & Wilks' lambda \\
\hline$\sigma_{\mathrm{r}}^{\prime}$ & 0.971 & 0.643 & 0.099 \\
$\sigma_{\theta}^{\prime}$ & 0.939 & 1.043 & 0.094 \\
$\sigma_{\mathrm{R}}^{\prime}$ & 0.858 & 0.085 & 0.106 \\
$\sigma_{\mathrm{C}}^{\prime}$ & 0.760 & 50.639 & 0.742 \\
$\sigma_{\text {dis }}^{\prime}$ & 0.760 & 5.844 & 0.180 \\
\hline
\end{tabular}

(2) Using the length-width ratio of the polygon $r$, the inclination direction $\theta$, rectangularity $R$, compactness $C$, and the center point $(x, y)$ as the characteristic parameters to carry out $K$-means clustering, one can efficiently divide irregular buildings into regular blocks. Then, by use of the weighted averages, shape dispersion parameters can be calculated to express the damage extent to individual buildings.
Among the shape dispersion parameters, at least $\sigma_{\theta}^{\prime}$, $\sigma_{\mathrm{R}}^{\prime}, \sigma_{\mathrm{C}}^{\prime}$, and $\sigma_{\text {dis }}^{\prime}$ values are suitable to reflect the extent of a building's damage. Higher values are indicative of more serious damage.

(3) On the basis of existing data collected in the Wenchuan earthquake zone, $\sigma_{\mathrm{C}}^{\prime}$ and $\sigma_{\text {dis }}^{\prime}$ were used to establish discrimination functions that can effectively distinguish the damage extent to buildings.

The TLS-BSAM is a new model by which laser scanning measurement technology can be used safely in the field to conduct building earthquake damage analysis. When combined with earthquake field survey work, the resulting information can be used as a valuable guide for assessments of the seismic intensity based on damage extents and prioritization of earthquake relief and disaster area reconstruction efforts. In addition, the obtained point cloud 


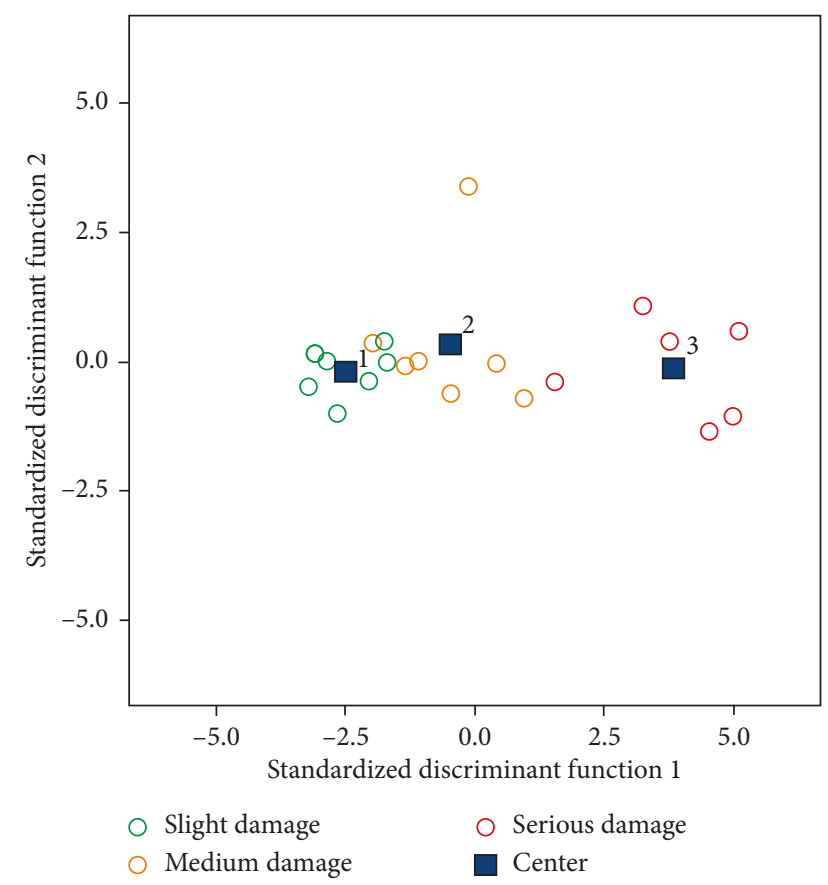

Figure 11: Fisher's linear discriminant results (function 1: $f(x)=$ $1.126 \sigma_{\mathrm{C}}^{\prime}+0.743 \sigma_{\mathrm{dis}}^{\prime}$; function 2: $\left.f(x)=-0.219 \sigma_{\mathrm{C}}^{\prime}+0.874 \sigma_{\mathrm{dis}}^{\prime}\right)$.

data provide important basic data for future building damage process numerical simulations and analyses. However, as the TLS-BSAM is a new model, it is still in the experimental stage. The model proposed in the paper is better used for buildings with structural or structural element damage. Presently, because of the few sample data points used in the experiments, the discriminant functions cannot yet be widely applied to building damage analyses in other areas. We plan on collecting more point cloud data for earthquake-damaged buildings to validate and improve the existing functions in the future, which would make them more widely applicable.

\section{Data Availability}

The TLS point cloud data used to support the findings of this study are available from the corresponding author upon request.

\section{Conflicts of Interest}

The authors declare that they have no conflicts of interest.

\section{Acknowledgments}

The authors thank all the developers of CloudCompare, which is a GPL three-dimensional point cloud and mesh processing software. This work was supported by the research grant from the Institute of Crustal Dynamics, China Earthquake Administration (grant no. ZDJ2017-29).

\section{References}

[1] K. Saito, R. J. S. Spence, C. Going, and M. Markus, "Using high-resolution satellite images for post-earthquake building damage assessment: a study following the 26 January 2001 Gujarat earthquake," Earthquake Spectra, vol. 20, no. 1, pp. 145-169, 2004.

[2] S. Stramondo, C. Bignami, M. Chini, N. Pierdicca, and A. Tertulliani, "Satellite radar and optical remote sensing for earthquake damage detection: results from different case studies," International Journal of Remote Sensing, vol. 27, no. 20, pp. 4433-4447, 2006.

[3] T. Balz and M. Liao, "Building-damage detection using postseismic high-resolution SAR satellite data," International Journal of Remote Sensing, vol. 31, no. 13, pp. 3369-3391, 2010.

[4] X. Lin, J. Zhang, X. Ning, M. Duan, and Y. Zang, "Filtering of point clouds using fusion of three types of primitives including points, objects and key points," Acta Geodaetica et Cartographica Sinica, vol. 45, no. 11, pp. 1308-1317, 2016.

[5] M. He, Q. Zhu, Z. Du, H. Hu, Y. Ding, and M. Chen, “A 3D shape descriptor based on contour clusters for damaged roof detection using airborne LiDAR point clouds," Remote Sensing, vol. 8, no. 3, p. 189, 2016.

[6] M. Li, L. Cheng, J. Gong et al., "Post-earthquake assessment of building damage degree using LiDAR data and imagery," Science in China Series E: Technological Sciences, vol. 51, no. 2, pp. 133-143, 2008.

[7] H. Ma, C. Yao, and S. Zhang, "Some technical issues of airborne LiDAR system applied to Wenchuan earthquake relief works," Journal of Remote Sensing, vol. 12, no. 6, pp. 925-932, 2008.

[8] Y. Shao, H. Gong, S. Wang, F. Zhang, and W. Tian, "Multisource SAR remote sensing data for rapid response to Wenchuan earthquake damage assessment," Journal of Remote Sensing, vol. 12, no. 6, pp. 865-870, 2008.

[9] X. Tong, Z. Hong, S. Liu et al., "Building-damage detection using pre- and post-seismic high-resolution satellite stereo imagery: a case study of the May 2008 Wenchuan earthquake," ISPRS Journal of Photogrammetry and Remote Sensing, vol. 68, pp. 13-27, 2012.

[10] A. Biasion, L. Bornaz, and F. Rinaudo, "Laser scanning applications on disaster management," in Proceedings of the 1st International Symposium on Geo-Information for Disaster Management (Gi4DM), Delft, Netherlands, March 2005.

[11] M. Alba, F. Roncoroni, and M. Scaioni, "Application of TLS for change detection in rock faces," International Archives of the photogrammetry, Remote Sensing and Spatial Information Sciences, vol. 38, no. 3, pp. 99-104, 2009.

[12] A. Abellán, J. Calvet, J. M. Vilaplana, and J. Blanchard, "Detection and spatial prediction of rockfalls by means of terrestrial laser scanner monitoring," Geomorphology, vol. 119, no. 3-4, pp. 162-171, 2010.

[13] N. A. Haddad, "From ground surveying to 3D laser scanner: a review of techniques used for spatial documentation of historic sites," Journal of King Saud University-Engineering Sciences, vol. 23, no. 2, pp. 109-118, 2011.

[14] F. Pirotti, A. Guarnieri, and A. Vettore, "Ground filtering and vegetation mapping using multi-return terrestrial laser scanning," ISPRS Journal of Photogrammetry and Remote Sensing, vol. 76, pp. 56-63, 2013.

[15] M. J. Olsen, K. F. Cheung, Y. Yamazaki et al., "Damage assessment of the 2010 Chile earthquake and tsunami using terrestrial laser scanning," Earthquake Spectra, vol. 28, no. 1, pp. S179-S197, 2012. 
[16] M. J. Olsen and R. Kayen, "Post-earthquake and tsunami 3D laser scanning forensic investigations," Forensic Engineering, pp. 477-486, 2012.

[17] S. Loncaric, "A survey of shape analysis techniques," Pattern Recognition, vol. 31, no. 8, pp. 983-1001, 1998.

[18] D. Zhang and G. Lu, "Review of shape representation and description techniques," Pattern Recognition, vol. 37, no. 1, pp. 1-19, 2004.

[19] F. P. Preparata and S. J. Hong, "Convex hulls of finite sets of points in two and three dimensions," Communications of the ACM, vol. 20, no. 2, pp. 87-93, 1977.

[20] R. L. Graham and F. Frances Yao, "Finding the convex hull of a simple polygon," Journal of Algorithms, vol. 4, no. 4, pp. 324-331, 1983. 


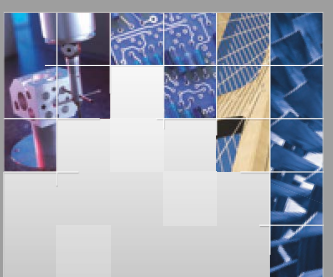

\section{Enfincering}
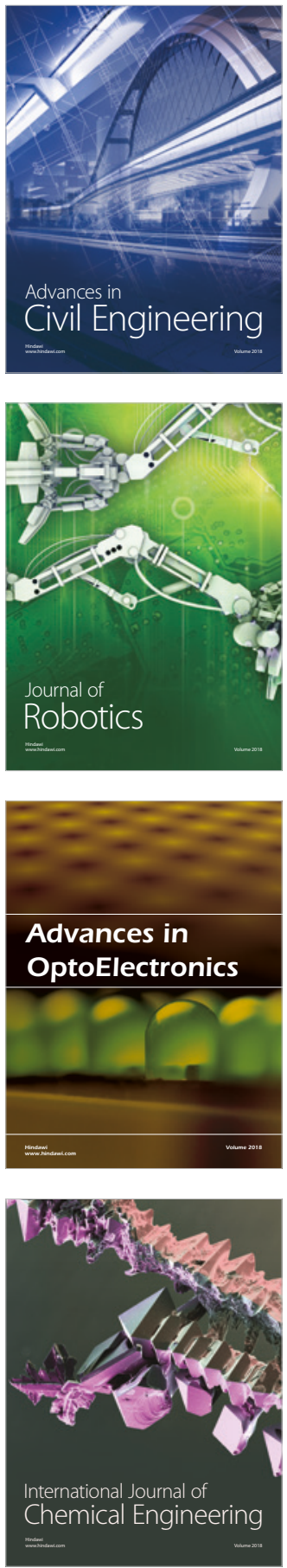

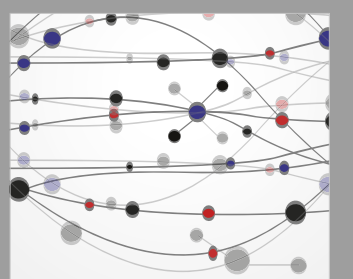

\section{Rotating \\ Machinery}

The Scientific World Journal

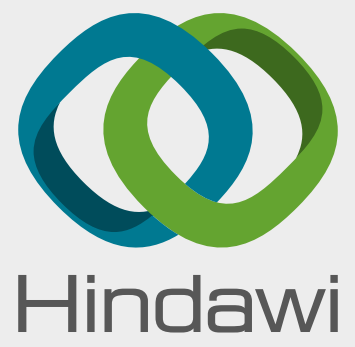

Submit your manuscripts at

www.hindawi.com
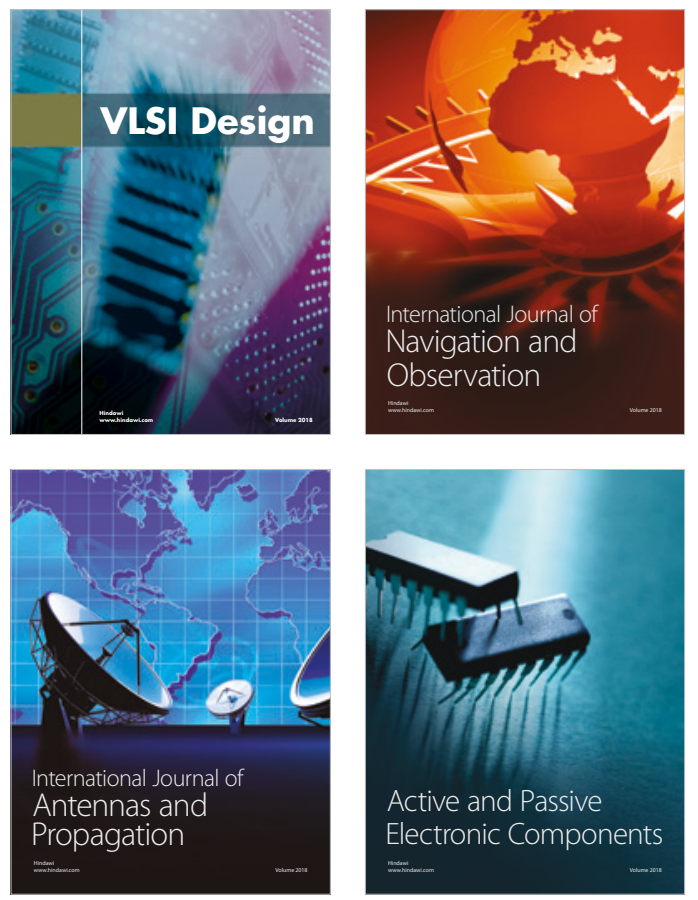
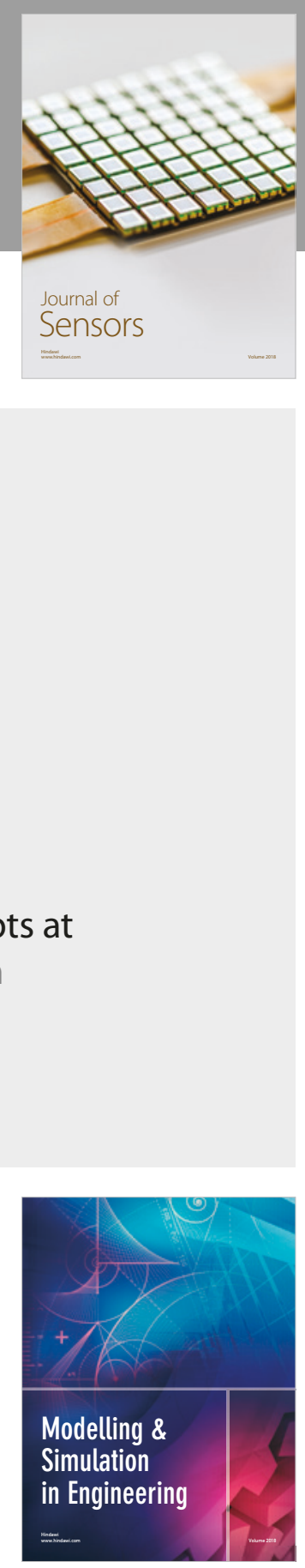

\section{Advances \\ Multimedia}
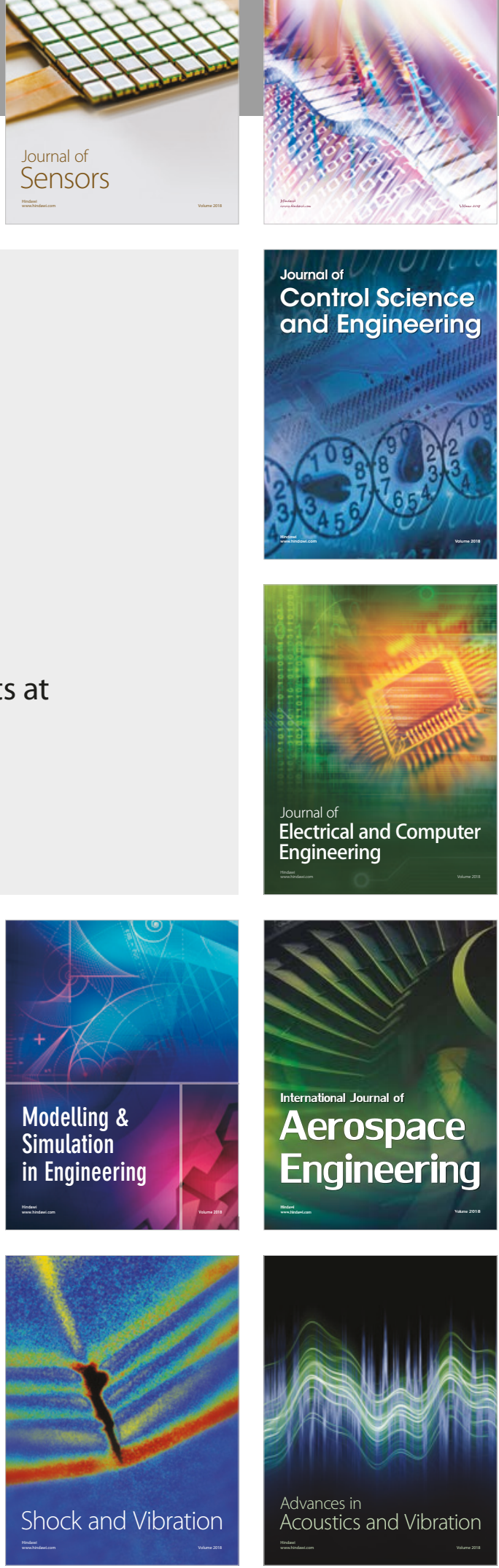